\title{
Cobalt (II), Nickel (II), and Copper (II) Complexes of Polystyrene-Supported Schiff Bases
}

\author{
N. R. Suja, K. K. Mohammed Yusuff \\ Department of Applied Chemistry, Cochin University of Science and Technology, Cochin 682022, India
}

Received 27 December 2002; revised 25 May 2003; accepted 10 August 2003

\begin{abstract}
Two novel polystyrene-supported Schiff bases, PSOPD and PSHQAD, were synthesized. A polymerbound aldehyde was condensed with o-phenylenediamine to prepare the Schiff base PSOPD, and a polymer-bound amine was condensed with 3-hydroxyquinoxaline-2-carboxaldehyde to prepare the Schiff base PSHQAD. This article addresses the study of cobalt (II), nickel (II), and copper (II) complexes of these polymer-bound Schiff bases. All the complexes were characterized, and the probable geometry was
\end{abstract}

suggested using elemental analysis, diffuse reflectance ultraviolet, Fourier transform infrared spectroscopy, thermal studies, surface area studies, and magnetic measurements. (C) 2004 Wiley Periodicals, Inc. J Appl Polym Sci 91: 3710-3719, 2004

Key words: polystyrene-bound Schiff base; o-phenylenediamine; 3-hydroxyquinoxaline-2-carboxaldehyde; polymermetal complexes; supported complexes

\section{INTRODUCTION}

Metal complexes are supported on polymers to give an organic polymer inorganic function. ${ }^{1}$ Such complexes may show a specific type of catalytic activity reflecting the properties of polymers. In metalloenzymes, such as hemoglobin and oxidase, where the metal complex is the active site, the organic part plays an important role in the reactivity. ${ }^{2-4}$ Such catalysts are used extensively now in emerging areas such as triphase catalysis. ${ }^{5}$ Schiff bases, an important class of ligands obtained by condensation of aldehyde with amine, play a significant role in such applications. ${ }^{6-8}$ We report herein the synthesis of cobalt (II) [Co(II)], nickel (II) [Ni(II)], and copper (II) [Cu(II)] complexes with the polymer-anchored Schiff bases I and II and their characterization using various physicochemical techniques.

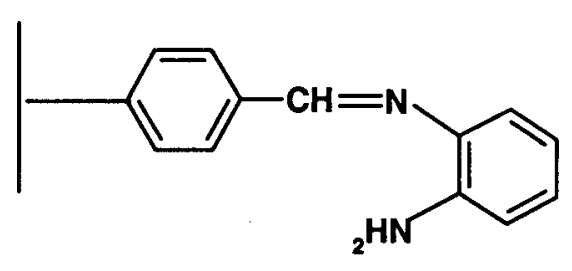

II

\section{EXPERIMENTAL}

Chloromethylated polystyrene $[3.1 \mathrm{mmol} \mathrm{Cl} / \mathrm{g}$ (Merck)] crosslinked with $2 \%$ divinyl benzene was used as the starting material. The amine-bound ${ }^{9}$ and the aldehyde-bound ${ }^{10}$ polystyrene was synthesized from the chloromethylated polystyrene according to the literature procedure. o-Phenylenediamine was

Correspondence to: K. K. Mohammed Yusheff (yusuff@ cusat.ac.in).

Journal of Applied Polymer Science, Vol. 91, 3710-3719 (2004) (C) 2004 Wiley Periodicals, Inc. purchased from CDH Laboratories and was purified by recrystallization from its hot solution, which was treated with activated charcoal. 3-Hydroxyquinoxaline-2-carboxaldehyde was prepared according to a reported procedure. ${ }^{11}$ All metal chlorides were Merck samples and were used as such.

\section{Synthesis of polymer-anchored schiff base of $o$-phenylenediamine}

The polymer-bound benzaldehyde (4 g) was swelled in ethanol for 1 hour. o-Phenylenediamine $(1.08 \mathrm{~g})$ was dissolved in ethanol $(50 \mathrm{~mL})$. This was then refluxed 
on a water bath for 6 hours. The resulting Schiff base, PSOPD, was filtered, washed with ethanol several times, and dried in vacuum over anhydrous calcium chloride.
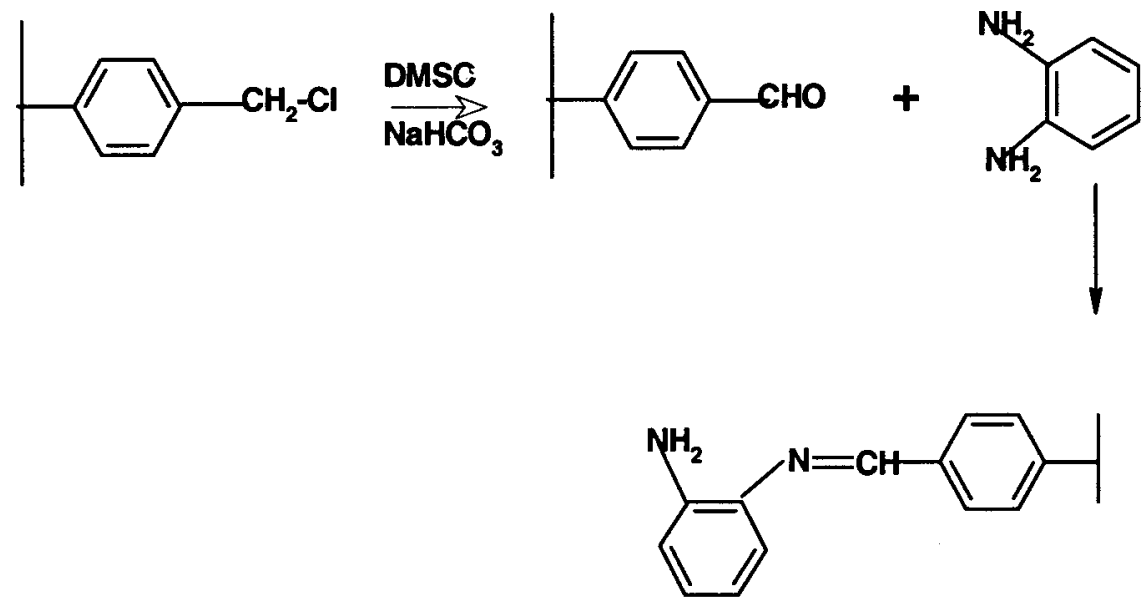

Synthesis of cobalt, nickel, and copper complexes

The polymer-bound Schiff base PSOPD ( $3 \mathrm{~g}$ ) was swelled in chloroform $(25 \mathrm{~mL})$ for 1 hour. It then was filtered and refluxed with a solution of the metal chloride $\left(1.70 \mathrm{~g}\right.$ of $\mathrm{CuCl}_{2} \cdot 2 \mathrm{H}_{2} \mathrm{O}, 2.37 \mathrm{~g}$ of $\mathrm{CoCl}_{2} \cdot 6 \mathrm{H}_{2} \mathrm{O}$, or $2.37 \mathrm{~g}$ of $\mathrm{NiCl}_{2} \cdot 6 \mathrm{H}_{2} \mathrm{O}$ in $100 \mathrm{~mL}$ ethanol) on a water bath for approximately 6 hours. All these complexes then were washed several times with ethanol and dried in vacuum over anhydrous calcium chloride.

Synthesis of polymer-anchored schiff base of 3-hydroxyquinoxaline-2-carboxaldehyde

3-Hydroxyquinoxaline-2-carboxaldehyde [500 $\mathrm{mL}$ of $0.005 \%(\mathrm{w} / \mathrm{v})]$ solution was refluxed with polymer- bound amine $(5 \mathrm{~g})$ for approximately 8 hours. The resulting Schiff base was filtered and washed with water and alcohol and dried in vacuum over anhydrous calcium chloride.

\section{Synthesis of CO(II), NI(II), and CU(II) complexes}

The polymer-bound Schiff base PSHQAD ( $3 \mathrm{~g}$ ) was swelled in chloroform for 1 hour. After swelling, it was filtered and refluxed with a solution metal chloride $1.70 \mathrm{~g}$ of $\mathrm{CuCl}_{2} 2 \mathrm{H}_{2} \mathrm{O}, 2.37 \mathrm{~g}$ of $\mathrm{CoCl}_{2} 6 \mathrm{H}_{2} \mathrm{O}$, or $2.37 \mathrm{~g}$ of $\mathrm{NiCl}_{2} 6 \mathrm{H}_{2} \mathrm{O}$ in $100 \mathrm{~mL}$ ethanol) for approximately 6 hours. The complexes thus formed were washed with ethanol several times, filtered, and dried in vacuum over anhydrous calcium chloride.

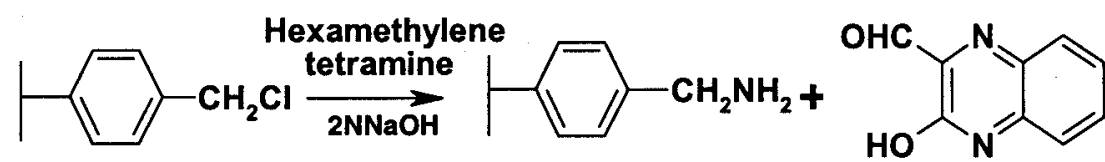

\section{Analytical methods}

Microanalysis for $\mathrm{C}, \mathrm{H}$, and $\mathrm{N}$ were carried out on a Perkin Elmer 2400 elemental analyzer. Metal analyses were carried out by ICP measurements after digesting the samples in Conc. $\mathrm{HNO}_{3}$. The percentage of chlo-

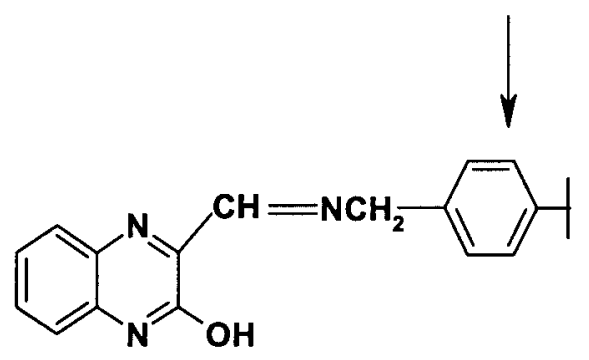

rine in the sample was determined using the Volhards method after digesting the samples in pyridine. ${ }^{12}$ Magnetic susceptibility of the complexes was determined at RT using the Gouy method, using Co$\left[\mathrm{Hg}(\mathrm{NCS})_{4}\right]$ as standard. Electronic spectra were obtained using a Cary Win UV-Vis spectrophotometer in 
TABLE I

Elemental Analysis Data of the Ligand and Polymer-Bound Complexes

\begin{tabular}{lccccc}
\hline Compound & $\% \mathrm{C}$ & $\% \mathrm{H}$ & $\% \mathrm{~N}$ & $\% \mathrm{M}$ & $\% \mathrm{CI}$ \\
\hline PSOPD & 77.76 & 7.36 & 3.32 & - & - \\
CoPSOPD & 74.66 & 6.58 & 2.88 & 2.2 & 2.5 \\
NiPSOPD & 77.59 & 7.11 & 2.48 & 1.5 & - \\
CuPSOPD & 74.45 & 7.04 & 2.61 & 1.5 & - \\
PSHQAD & 77.57 & 6.80 & 4.43 & - & - \\
CoPSHQAD & 72.36 & 6.70 & 3.00 & 2.3 & 3 \\
NiPSHQAD & 76.35 & 6.79 & 3.77 & 1.3 & 3 \\
CuPSHQAD & 73.91 & 6.73 & 3.31 & 1.1 & - \\
\hline
\end{tabular}

the diffuse reflectance mode at RSIC, IIT, Chennai. Infrared spectra of the complexes were taken in region $400-4000 \mathrm{~cm}^{-1}$ as $\mathrm{KBr}$ discs using a Shimadzu 8100 FTIR spectrophotometer. The ESR spectra of the complexes were recorded at liquid $\mathrm{N}_{2}$ temperature using E-109X/Q band ESR spectrometer. The calibration was carried out using DPPH (1,1, diphenyl-2-picrylhydrazyl) as the field marker. Surface area measurements were carried out using the BET method using a Micromeritics Gemini 2360 surface area analyzer. Thermogravimetric analysis was carried out on a Shi-
TABLE II

EPR and Magnetic Moment Data for the Complexes

\begin{tabular}{llcc}
\hline Complexes & $g_{\perp}$ & $g_{\text {II }}$ & $\begin{array}{c}\text { Magnetic } \\
\text { moment (BM) }\end{array}$ \\
\hline CoPSHQAD & 2.20 & 2.70 & 2.2 \\
NiPSHQAD & 2.023 & & 3.0 \\
CuPSHQAD & 2.07 & 2.27 & 2.1 \\
CoPSOPD & 2.004 & 2.20 & 2.5 \\
NiPSOPD & 2.004 & 2.076 & 3.3 \\
CuPSOPD & 2.06 & 2.20 & 1.8 \\
\hline
\end{tabular}

madzu TGA-50 at a heating rate of $20^{\circ} \mathrm{C}$ under $\mathrm{N}_{2}$ atmosphere.

\section{RESULTS AND DISCUSSION}

The chloromethylated polystyrene containing approximately $13 \% \mathrm{Cl}$, the starting material for the preparation of Schiff base, on treatment with dimethyl suphoxide and sodium bicarbonate yielded polymerbound benzaldehyde, which was evidenced by the positive test with Borsche's reagent. The chlorine content of the polymer-bound aldehyde was negligible,

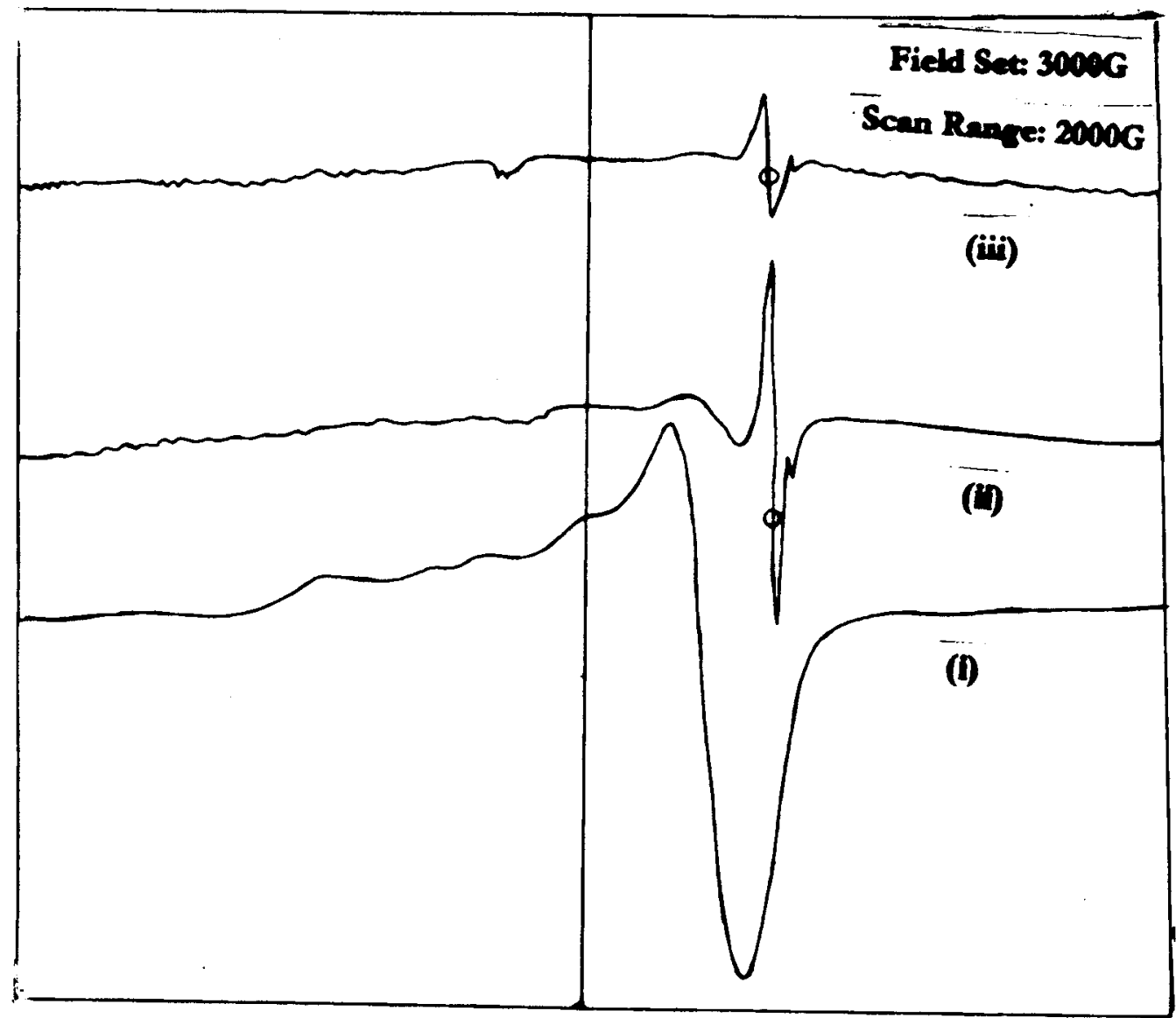

Figure 1 EPR spectra of Polymer bound OPD complexes i) CuPSOPD ii) NiPSOPD iii) CoPSOPD. 


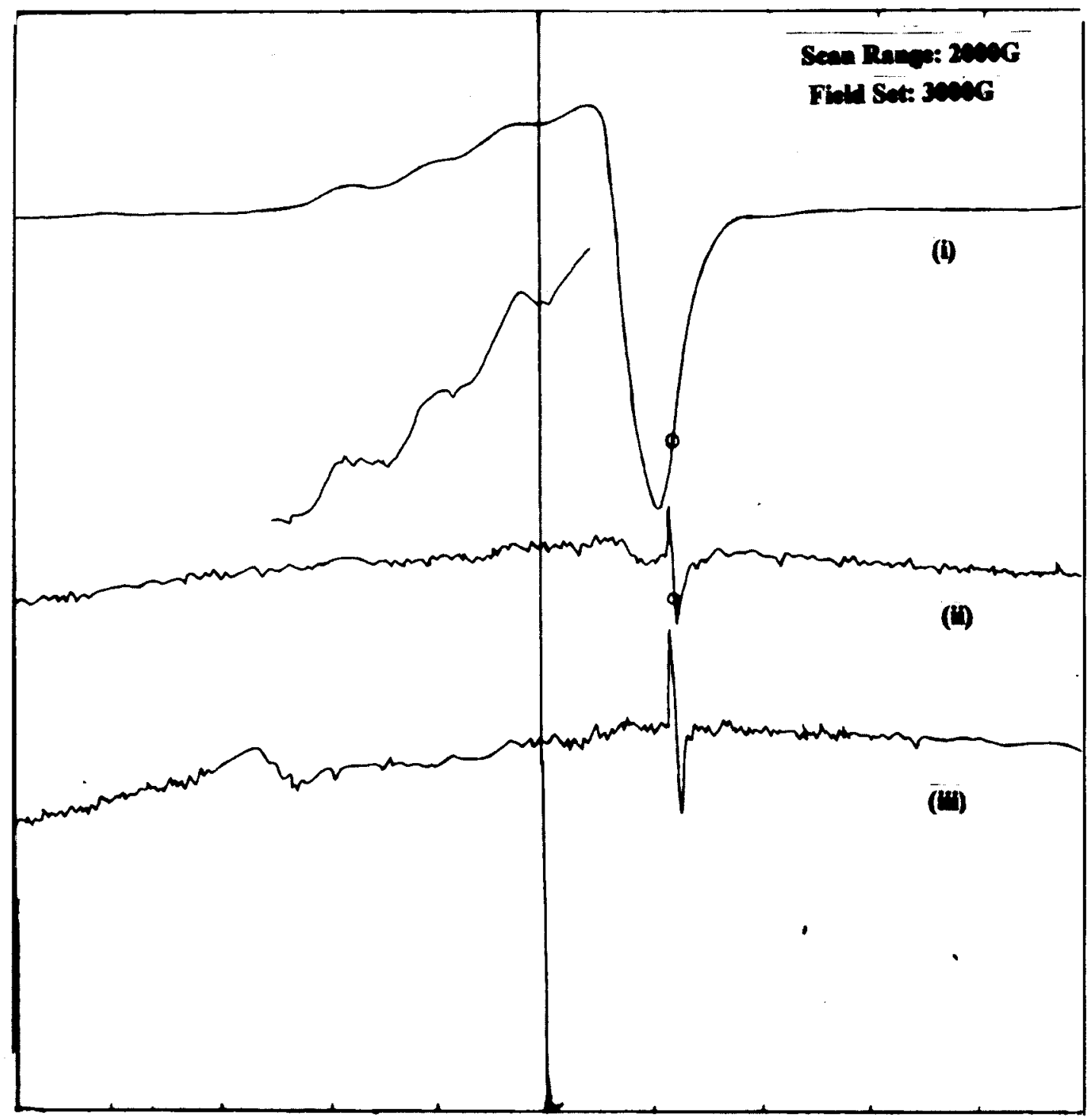

Figure 2 EPR spectra of complexes i) CUPSHQAD ii) NiPSHQAD iii) CoPSHQAD.

which indicates that almost all chloromethylene groups are converted into aldehyde. Aminomethyl polystyrene prepared by the polymer analog of delepine reaction as indicated in the scheme above gave

TABLE III

Thermogravimetric Analysis Data of PSHQAD and Metal Complexes

\begin{tabular}{lcc}
\hline Compound & Temperature Range & \% Weight Loss \\
\hline PSHQAD & $110-405$ & 39.5 \\
& $405-600$ & 53.7 \\
CoPSHQAD & $110-406$ & 38.4 \\
& $406-500$ & 47.5 \\
NiPSHQAD & $110-405$ & 41.6 \\
& $405-538$ & 47.2 \\
CuPSHQAD & $110-385$ & 41.1 \\
& $385-447$ & 48.2 \\
\hline
\end{tabular}

a positive test for the presence of amino group (a deep blue color with Ninhydrin reagent). The percentage of nitrogen indicates that there is only $14 \%$ conversion. The nitrogen percentage in the Schiff base indicates that the azomethine group units are approximately 7 to 8 units apart. The metal binding capacity was found to be highest for cobalt. The porous structure of the polymer seems to be blocked, which is indicated by the drastic decrease in surface area from 154 to 200 $\mathrm{m}^{2} / \mathrm{g}$ to approximately 45 to $50 \mathrm{~m}^{2} / \mathrm{g}$ in the case metal complexes.

\section{Magnetic susceptibility measurements}

For calculation of magnetic susceptibility, the Guoy method was used. Magnetic susceptibility of all the complexes showed a negative value, probably the 
TABLE IV

Thermogravimetric Analysis Data of PSOPD and Metal Complexes

\begin{tabular}{lcc}
\hline Compound & Temperature range & \% Weight loss \\
\hline PSOPD & $265-600$ & 25 \\
& $600-800$ & 48 \\
CoPSOPD & $376-424$ & 25 \\
& $424-500$ & 15 \\
& $500-800$ & 42 \\
NiPSOPD & $376-425$ & 20 \\
& $425-500$ & 20 \\
CuPSOPD & $500-800$ & 30 \\
& $378-413$ & 22 \\
& $413-457$ & 20 \\
& $457-800$ & 20 \\
\hline
\end{tabular}

result of the low concentration of the metal and the very large diamagnetic susceptibility of the atoms present in the polymer complex. However, the approximate molecular weight and empirical formula of the repeating complex unit containing one metal atom was calculated using a reported procedure. ${ }^{13}$ The magnetic moments $\mu_{\text {eff }}$ of the different complexes calculated on the basis of these values are given in the Table II. The cobalt complex Co(II)PSOPD has a magnetic moment of 2.5 BM. Magnetic moment values of 2.0 to 2.5 usually are reported for square planar $\mathrm{Co}(\mathrm{II})$ complexes. This higher magnetic moment value (when compared with that expected for one unpaired electron) for low-spin square planar complexes corresponds to the spinonly value for one unpaired electron multiplied by a factor of $[1+(2 \lambda / \delta)]$. Here, $\lambda$ is the single electron spin-orbit coupling constant, and $\delta$ is the separation between the $d_{x y}$ orbit and its neighboring levels. Such high values are reported for square planar complexes like $\mathrm{Co}\left(\mathrm{PEt}_{3}\right)_{2}(\mathrm{NCS})_{2}$, which has a magnetic moment value of $2.3 \mathrm{BM} .{ }^{14}$ Cobalt complexes of the ligand Salen also are planar, with a magnetic moment value of 2.2 BM. Thus, the present $\mathrm{Co}$ (II)PSOPD complex can be considered to have a square planar structure. Ni(II)PSOPD has a $\mu_{\text {eff }}$ of $3.3 \mathrm{BM}$, which suggests an octahedral structure, and $\mathrm{Cu}(\mathrm{II})-$ PSOPD has a magnetic moment of $1.8 \mathrm{BM}$, indicating a distorted octahedral structure.

Among the complexes of PSHQAD, the Co(II)PSHQAD has a magnetic moment of $2.2 \mathrm{BM}$, indicat- ing a square pyramidal geometry for the complex. The $\mathrm{Ni}(\mathrm{II}) \mathrm{PSHQAD}$ exhibits a magnetic moment value of 3.0 BM, which is suggestive of an octahedral structure. $\mathrm{Cu}(\mathrm{II}) \mathrm{PSHQAD}$ exhibits a slightly higher magnetic moment value of $2.1 \mathrm{BM}$, indicating a tetrahedral geometry for this complex.

\section{EPR spectra}

EPR spectra of the complexes were recorded at liquid nitrogen temperature. EPR parameters of the complexes are given in the Table II.

All the complexes of PSOPD are seen to be EPR active (Figure 1). For the Co(II)PSOPD complex, $g_{\text {II }}$ and $g_{\perp}$ values are found to be 2.004 and 2.20, respectively. These values suggest a square planar structure. The assignment of this geometry is supported by the magnetic moment value of $2.5 \mathrm{BM}$. The Ni(II)PSOPD is EPR active, and $g_{\perp}$ and $g_{\text {II }}$ values for this complex are 2.004 and 2.076, respectively. ${ }^{15}$ This suggests an octahedral structure for this complex. The EPR spectrum of the polymeranchored $\mathrm{Cu}(\mathrm{II}) \mathrm{PSOPD}$ complex shows two $g$ values $\left(g_{\mathrm{II}}=2.28\right.$ and $\left.g_{\perp}=2.08\right)$, indicating a tetragonal symmetry or a distorted octahedral structure. The $A_{\text {II }}$ and $A_{\perp}$ values for $\mathrm{Cu}(\mathrm{II}) \mathrm{PSOPD}$ are found to be $150 \times 10^{-4} \mathrm{~cm}^{-1}$ and $66 \times 10^{-4} \mathrm{~cm}^{-1}$.

The EPR spectra of all the complexes of PSHQAD are depicted in Figure 2. The EPR spectrum of Co(II)PSHQAD gives two $g$ values, $g_{\text {II }}=2.70$ and $g_{\perp}=2.02$, indicating a square pyramidal structure. The EPR spectrum exhibiting a $g$ value of 2.023 was observed in the case of Ni(II)PSHQAD. This may be the result of the octahedral structure for the complex. The $\mathrm{Cu}(\mathrm{II})-$ PSHQAD complex also exhibits two $g$ values, $g_{\text {II }}$ $=2.26$ and $g_{\perp}=2.06$, and the $A_{\mathrm{II}}$ and $A_{\perp}$ values are $133 \times 10^{-4} \mathrm{~cm}^{-1}$ and $60 \times 10^{-4} \mathrm{~cm}^{-1}$.

The polymer-anchored CU(II) complexes exhibit a covalent environment as indicated by a $g_{\text {II }}$ value less than 2.3. ${ }^{16}$ The strength of the ligand can be assessed from the $G$ value, which can be calculated using the following equation ${ }^{17}$ :

$$
G=\frac{g_{\text {II }}-2.002}{g_{\perp}-2.002}
$$

If it is less than 4, the ligand forming the CU(II) complex is regarded as a strong field ligand. In the

TABLE V

FTIR Spectral Data (in $\mathrm{cm}^{-1}$ ) of PSOPD and Metal Complexes

\begin{tabular}{lccccc}
\hline Compound & $\begin{array}{c}\text { N-H } \\
\text { stretching }\end{array}$ & $\begin{array}{c}\text { Azomethine } \\
\text { C=N stretching }\end{array}$ & C-N stretching & M-N stretching \\
\hline PSOPD & 3436 & 1650 & 1268 & 1162 & \\
CoPSOPD & 3436 & 1635 & 1275 & 1175 & 552 \\
NiPSOPD & 3436 & 1640 & 1275 & 1175 & 539 \\
CuPSOPD & 3436 & 1626 & 1275 & 1175 & 565 \\
\hline
\end{tabular}




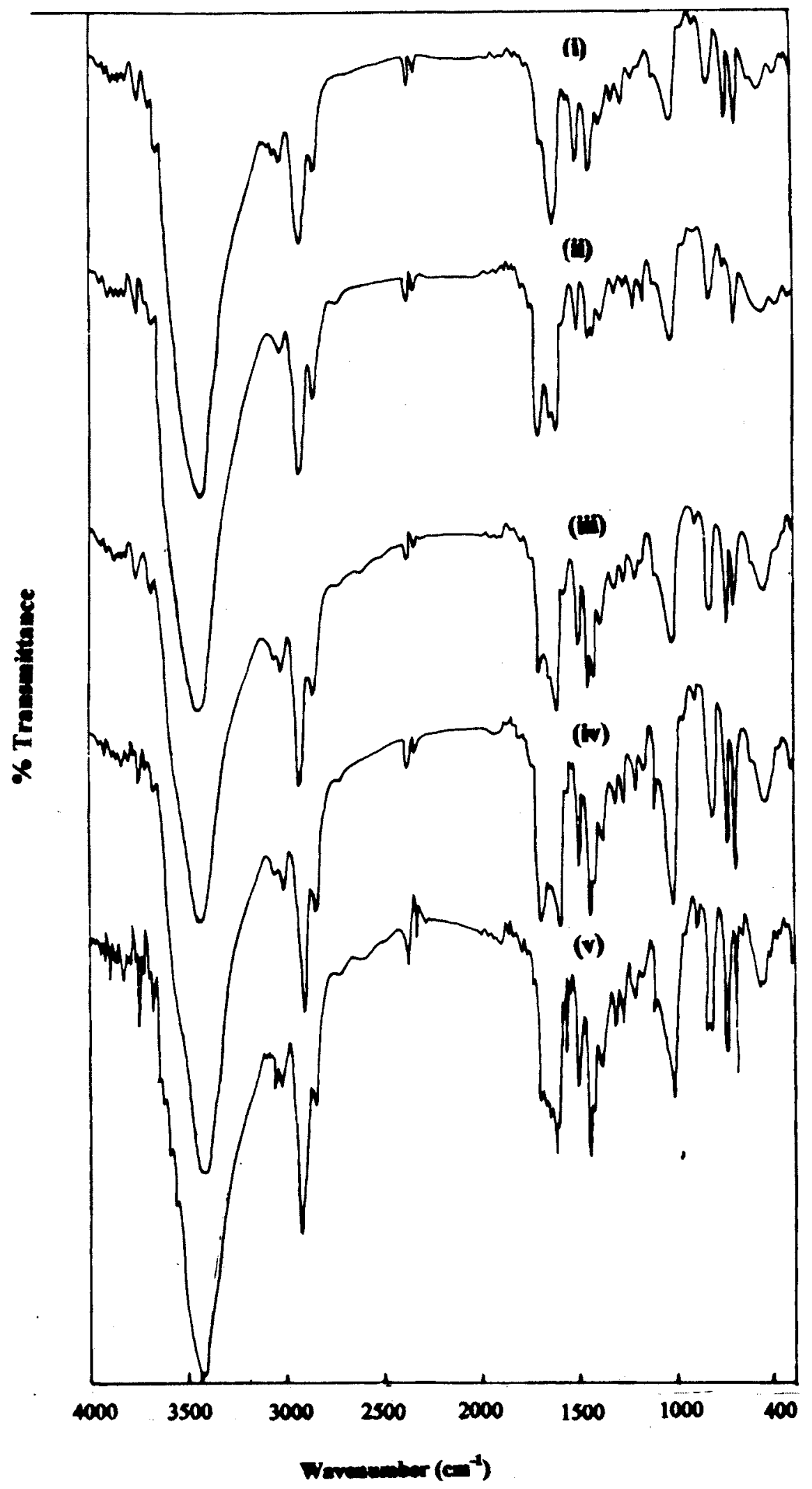

Figure 3 IR spectra of PSOPD complexes i) PSCHO ii) PSOPD iii) CoPSOPD iv) NiPSOPD v) CuPSOPD. 
TABLE VI

FTIR Spectral Data (in $\mathrm{cm}^{-1}$ ) of PSHQAD and the Metal Complexes

\begin{tabular}{lcccccc}
\hline Complex & $\begin{array}{c}\text { O-H } \\
\text { stretching }\end{array}$ & $\begin{array}{c}\text { Aliphatic } \\
\text { C-N } \\
\text { stretching }\end{array}$ & $\begin{array}{c}\text { C-O } \\
\text { stretching }\end{array}$ & $\begin{array}{c}\text { Azomethine } \\
\text { C=N } \\
\text { stretching }\end{array}$ & $\begin{array}{c}\text { Aldehydic } \\
\text { C-H } \\
\text { stretching }\end{array}$ & $\begin{array}{c}\text { Aromatic } \\
\text { ring C-N }\end{array}$ \\
\hline PSHQAD & - & 1029 & 1573 & 1660 & 2740 & 1460 \\
CoPSHQAD & 3549 & 1023 & 1560 & 1645 & 2727 & 1454 \\
NiPSHQAD & - & 1023 & 1566 & 1650 & 2733 & 1454 \\
CoPSHQAD & - & 1016 & 1566 & 1650 & 2727 & 1447 \\
\hline
\end{tabular}

present case, it was observed to be less than 4 [ $G$ value is 3.79 for $\mathrm{Cu}(\mathrm{II})$ PSHQAD and $\mathrm{G}=3.56$ for $\mathrm{Cu}$ (II)PSOPD], indicating the strong field nature of the ligands. The in-plane covalence parameter $\alpha^{2} \mathrm{Cu}$ was found to be 0.7676 and 0.6992 for $\mathrm{Cu}(\mathrm{II}) \mathrm{PSOPD}$ and $\mathrm{Cu}(\mathrm{II}) \mathrm{PSHQAD}$, respectively, which also supports covalent nature.

\section{Thermal analysis}

Thermal analysis of the PSHQAD complexes was carried out to find the stability of the complexes. All the complexes were stable up to $110^{\circ} \mathrm{C}$. At less than $100^{\circ} \mathrm{C}$, there appears to be a small decrease in weight corresponding to a weight loss of $4 \%$. This may be the result of the loss of physisorbed water. Therefore, the complexes can be considered to decompose in two stages (Table III). There was an approximately $40 \%$ weight loss during the second stage of decomposition. The decomposition of the Schiff base PSHQAD is almost complete at $600^{\circ} \mathrm{C}$, whereas the decomposition of the complexes was found to be complete at a lower temperature. The lower decomposition temperature in the case of the complexes may be the result of the catalytic activity of the metal ion in the decomposition of the polymer part.

The PSOPD complexes are stable up to $350^{\circ} \mathrm{C}$. Up to $350^{\circ} \mathrm{C}$, there is only an $8 \%$ weight loss resulting from adsorbed water or solvent molecules. Further decomposition of the complexes occurs in three stages (Table IV). Among the complexes, the cobalt complex, $\mathrm{Co}(\mathrm{II})$ PSOPD, was found to be of lower stability. It may be seen that the PSOPD complexes were more stable than the PSHQAD complexes.

\section{Infrared spectra}

Infrared spectra of the ligands and the polymersupported complexes were taken as $\mathrm{KBr}$ pellets in the region of 400 to $4000 \mathrm{~cm}^{-1}$. The infrared frequencies of PSOPD and its complexes are given in Table V, and the spectra are depicted in Figure 3. It is seen that PSOPD exhibits a band at $1650 \mathrm{~cm}^{-1}$, which can be assigned to the azomethine ${ }^{18} \mathrm{C}=\mathrm{N}$ linkage of the Schiff base. This band is observed to show a negative shift by 10 to $25 \mathrm{~cm}^{-1}$ in the complexes, indicating the coordination of this nitrogen to the metal in the complexes. The bands at 1268, $1162 \mathrm{~cm}^{-1}$ resulting from stretching of the $\mathrm{C}-\mathrm{N}$ bond (the bond between the aromatic ring and the free amino group) seem to be shifted to a higher frequency. This is probably the result of the shift in electron density from the benzene ring through resonance as the nitrogen is coordinated to the metal. The bands, observed at $552 \mathrm{~cm}^{-1}$ for the cobalt complex, at $539 \mathrm{~cm}^{-1}$ for the nickel complex, and at $569 \mathrm{~cm}^{-1}$ for the copper complex, may be the result of $\mathrm{M}-\mathrm{N}$ stretching modes.

The spectral data for PSHQAD and its complexes are given in Table VI, and the spectra are represented in Figure 4. In the case of PSHQAD complexes also, the azomethine band at $1660 \mathrm{~cm}^{-1}$ for PSHQAD undergoes a red shift on complexation, indicating the coordination of this group. There is an additional peak at $3549 \mathrm{~cm}^{-1}$ for Co(II)PSHQAD resulting from $\mathrm{O}-\mathrm{H}$ stretching. The hydroxyl proton may be retained in this case, whereas in all the other cases, $\mathrm{O}-\mathrm{H}$ group is deprotonated during the complex formation. The $\mathrm{C}-\mathrm{O}$ stretching frequencies at $1573 \mathrm{~cm}^{-1}$ shifted toward a lower frequency, indicating the coordination of oxygen to the metal. The $\mathrm{C}-\mathrm{N}$ stretching of the aromatic ring nitrogen at 1460 $\mathrm{cm}^{-1}$ also shows a shift toward lower frequencies. This may be the result of the shift in the electron density from the quinoxaline ring as a result of the coordination of oxygen and nitrogen to the metal. The metal oxygen stretching frequencies are at 691, 590 , and $698 \mathrm{~cm}^{-1}$ for cobalt, nickel, and copper complexes, respectively.

\section{Diffuse reflectance ultraviolet spectra}

Because the polymer samples are insoluble in solvents, the spectra were recorded in the diffuse reflectance mode. ${ }^{19}$ The diffuse reflectance spectrum is a plot of \%R versus wavelength. A Kubelka Munk analysis was performed on the reflectance data.

The diffuse reflectance spectra of PSOPD complexes are presented in Figure 5. Copper complexes of 


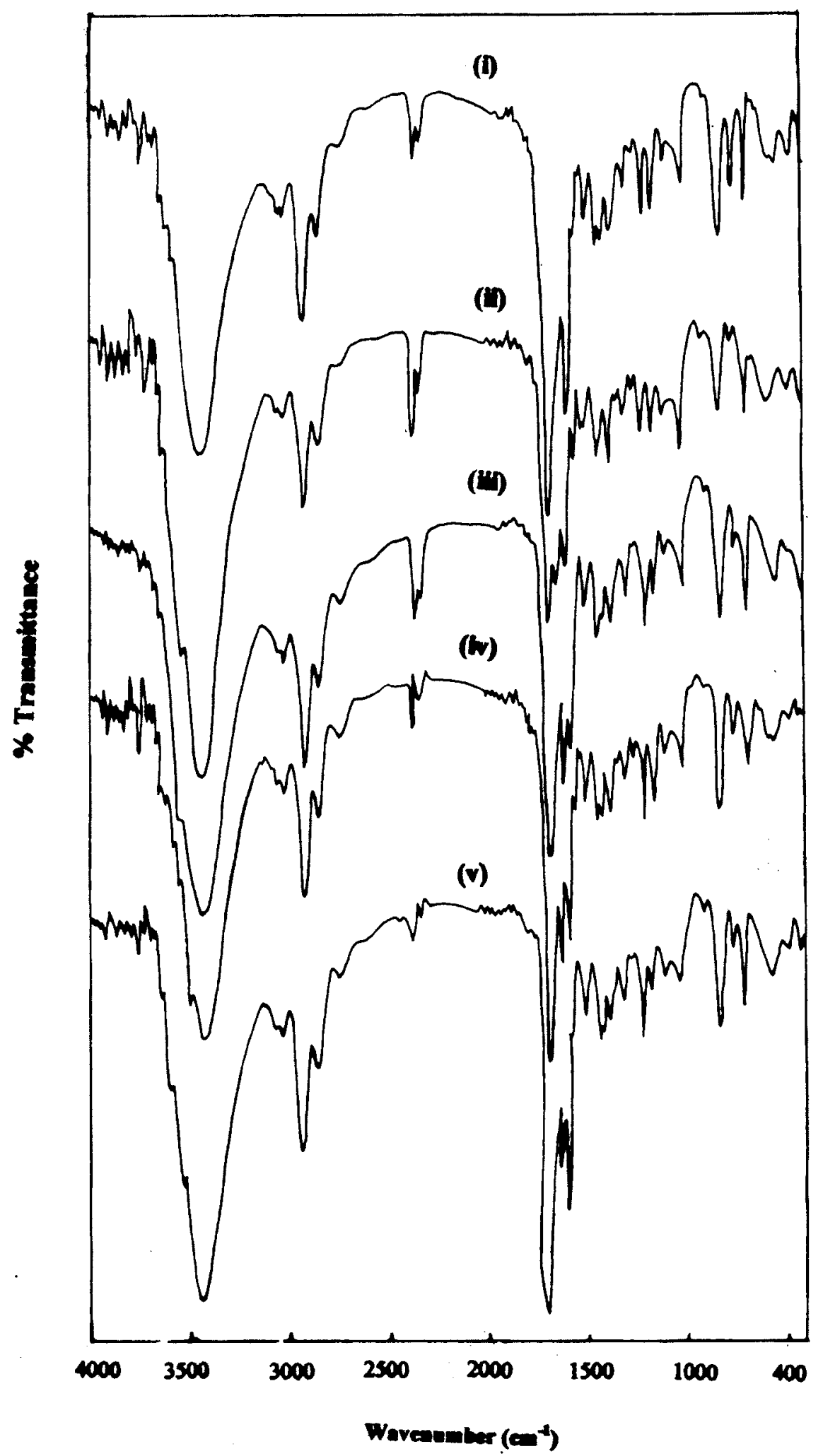

Figure 4 IR spectra of PSHQAD complexes i) $P_{2} N_{2}$ ii) PSHQAD iii) CoPSHQAD iv) NiPSHQAD v) CuPSHQAD.

PSOPD exhibit bands at 9500, 8300, 13,330 $\mathrm{cm}^{-1}$. A copper complex, $\mathrm{Cu}(\text { bipy })_{2}(\mathrm{ONO})^{+}$having absorption maxima at 9500 and $14,000 \mathrm{~cm}^{-1}$, has been reported to have a cis-distorted octahedral structure. ${ }^{20}$ The present copper complex may have assigned such a structure. The complex, Co(II)PSOPD, exhibits bands characteristic of square planar complexes at $8000 \mathrm{~cm}^{-1}$ and $11,500 \mathrm{~cm}^{-1}$. The complex is also EPR active. The 


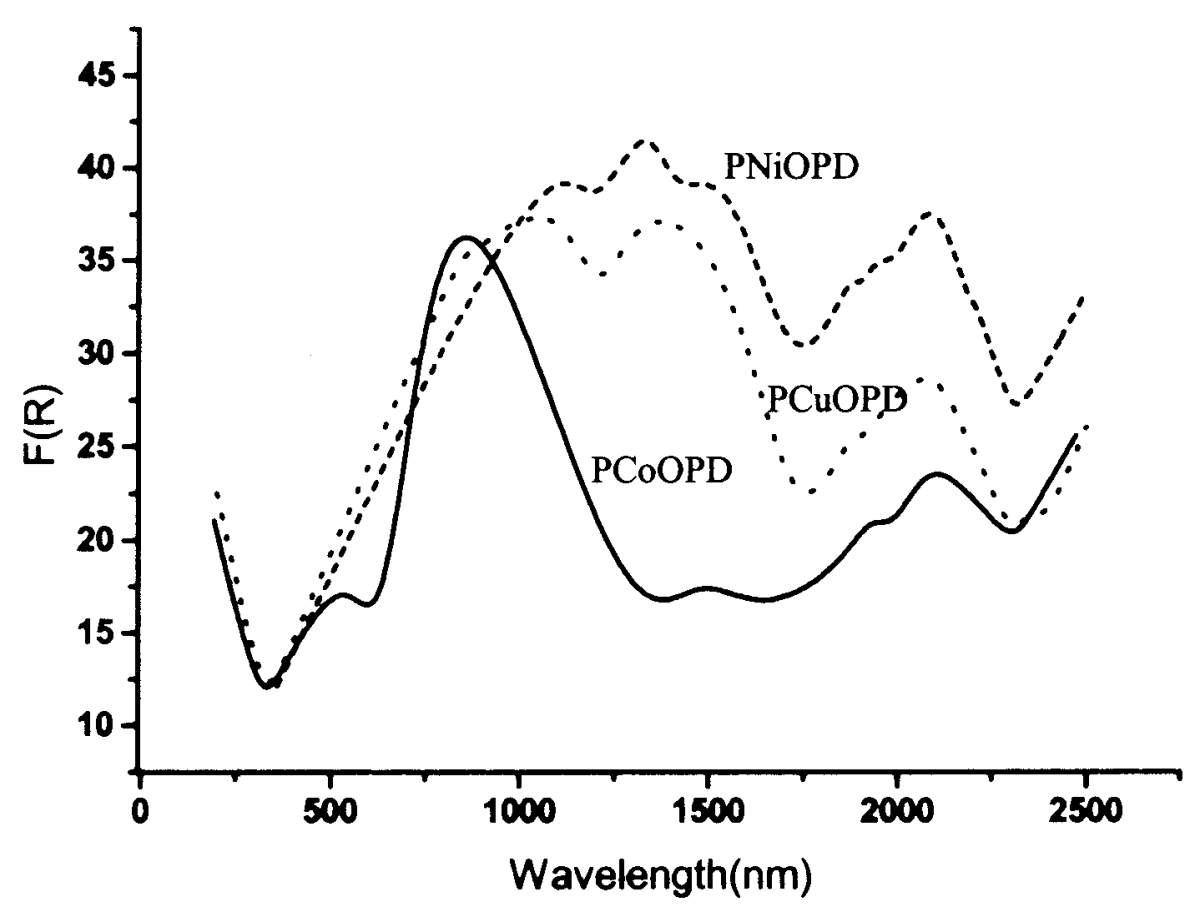

Figure 5 Diffuse reflectance spectra of PS-OPD complexes.

nickel complex showed bands at 8000 and 13,000 $\mathrm{cm}^{-1}$, suggesting octahedral structure.

Among the complexes of PSHQAD, the copper complex exhibits bands (Figure 6) at 16,000, 10,500, 9500,8000 , and $5000 \mathrm{~cm}^{-1}$, indicating a pseudotet- rahedral complex reported. ${ }^{21}$ The nickel complex exhibits bands at $9500 \mathrm{~cm}^{-1}$ and $12,000 \mathrm{~cm}^{-1}$, characteristic of a tetragonally distorted octahedral nickel complex. The cobalt complexes exhibit bands at $5300,8000,10,500,13,300$, and $17,000 \mathrm{~cm}^{-1}$, indi-

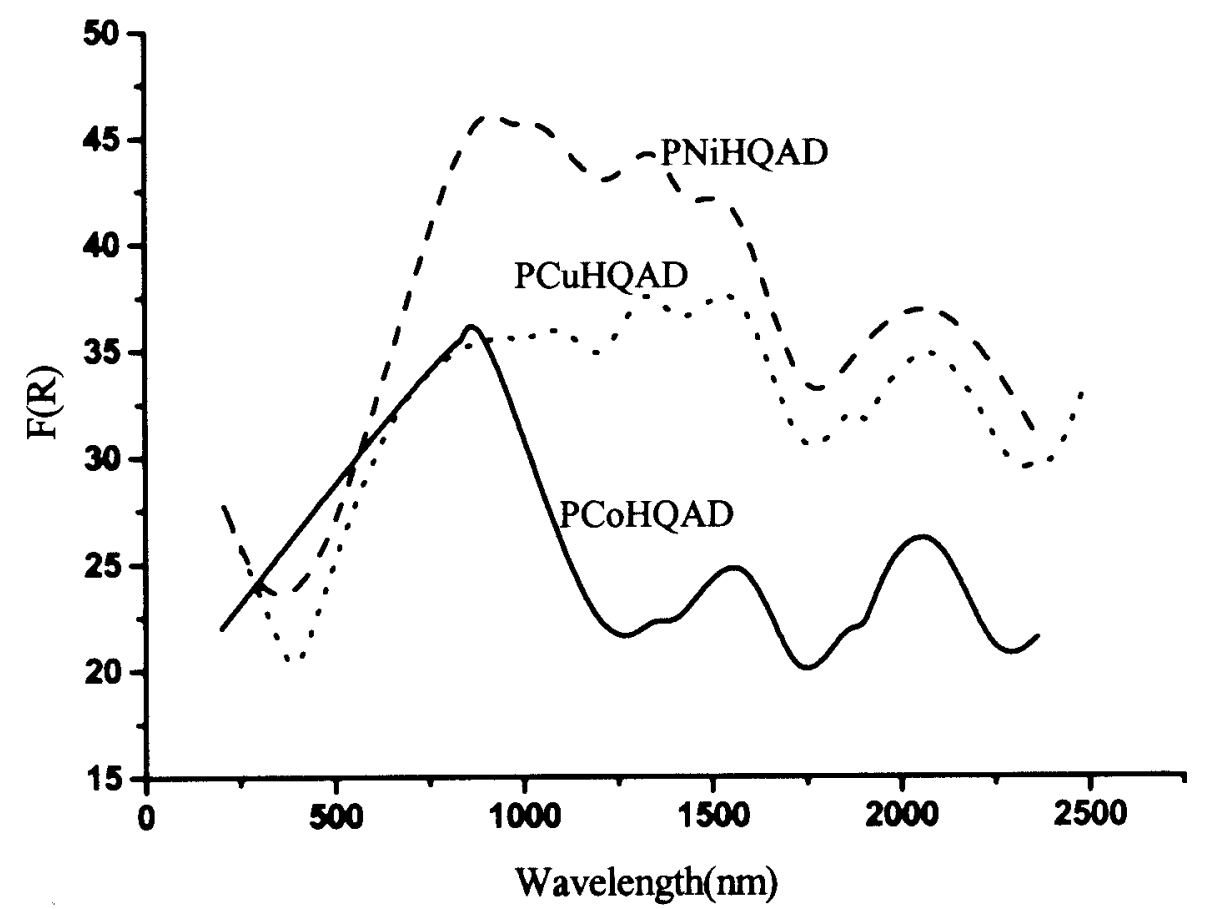

Figure 6 Diffuse reflectance spaectra of PS-HQAD complex. 
cating low-spin square pyramidal geometry. The complex is EPR active and has a magnetic moment value agreeing with this geometry.

The cobalt complex of Schiff-base PSHQAD is found to have square planar geometry. Such square planar complexes are found to act as catalysts in a number of reactions through reversible binding of oxygen. Thus, a series of Schiff-base complexes, which have the potential of being used as catalysts, were synthesized and were characterized using the above techniques.

One of the authors (SNR) thanks CSIR, New Delhi, India, for the award of Junior Research fellowship.

\section{References}

1. Tsuchida, E.; Nishide, N. Adv Polym Sci 1977, 24, 1.

2. Wohrle, D. Adv Polym Sci 1983, 50, 47.

3. Hughes, M. N. The Inorganic Chemistry of Biological Process, 2nd ed; Wiley: Chichester, 1981.

4. Hay, R. W. Bioinorganic Chemistry; Horwood: England, 1984.

5. Sasson, V.; Newmann, R. Handbook of Phase Transfer Catalysis; Chapmann and Hall: New York, 1997.
6. Bailer Jr, J. C. Catal Rev Sci Eng 1974, 10, 17

7. Holm, R. H.; Everett Jr, G. W.; Chakravorthy, A. Prog Inorg Chem 1968, 7, 83.

8. Syamal, A.; Maurya, M. R. Coord Chem Rev 1989, 95, 183.

9. Freuchet, J. M. J.; Hague, K. E. Macromolecules 1975, 8, 130.

10. Devaki, K. S.; Pillai, V. N. R. Eur Polym J 1988, 24, 209.

11. Mathew, J. Ph.D. Dissertation, Cochin University of Science and Technology, 1995.

12. Jeffery, G. H.; Bassett, J.; Mendham, J.; Denney, R. C. Vogel's Textbook of Quantitative Inorganic Analysis, 5th ed.; ElbsLongman: Singapore, 1991.

13. Syamal, A.; Singh, M. M. Ind J Chem 1993, 32A, 42.

14. Melson, G. A.; Figgis, B. N. Transition Metal Chemistry; Marcel Dekker: New York, 1982; Vol. 8, 118.

15. Turco, A.; Pecile, C.; Nicolini, M.; Martelli, M. J Am Chem Soc 1963, 85, 3510.

16. Kivelson, D.; Neuman, R. J Chem Phy 1961, 35, 149.

17. Proctor, I. M.; Hathaway, B. J.; Nicholls, P. J Chem Soc A 1968, 1678.

18. Yusuff, K. K. M; Sreekala, R. J Polym Sci Part A Polym Chem 1992, 30, 2595.

19. Lever, A. B. P. Inorganic Electronic Spectroscopy; Elsevier: Amsterdam, 1984.

20. Fitzgerald, W.; Murphy, B.; Tyagi, S.; Walsh, B.; Hathaway, B. J. J Chem Soc Dalton Trans 1981, 271.

21. Ferguson, J. J Chem Phy 1964, 40, 3406. 